\title{
Full-field analysis of a planar anisotropic layered half-plane for concentrated forces and edge dislocations
}

\author{
By Chien-Ching M a And R U-Li Lin \\ Department of Mechanical Engineering, National Taiwan University, \\ Taipei, Taiwan 10617, Republic of China
}

Received 6 March 2001; accepted 26 February 2002; published online 8 August 2002

The problem of a planar anisotropic elastic layered half-plane subjected to concentrated forces and edge dislocations applied either in the layer or in the half-plane is analysed. One of the objectives of this study is to develop an effective analytical methodology to construct the exact full-field solution for this problem. By using the Lekhnitskii formalism for anisotropic elastic material with the Fourier-transformation technique, the explicit closed-form solutions for stresses in the layer and the halfplane are obtained. The solutions are suitable for loadings that are acting on the free surface or at the interface. The complete solutions for this problem consist only of the simplest solutions obtained from an infinite homogeneous medium with concentrated forces and edge dislocations. The solutions include Green's function for applied loadings in an infinite medium and an infinite number of image singularities that are induced to satisfy the boundary and interface conditions. It is shown that the physical meaning of the solution is the image method. The magnitudes and locations of image singularities are determined automatically from the mathematical method presented in this study. Numerical results for the full-field stress distribution in the layered half-plane medium subjected to concentrated forces or edge dislocations are discussed in detail.

Keywords: anisotropic elastic material; layered half-plane;

Fourier transformation; image method

\section{Introduction}

Among the formalisms for two-dimensional linear anisotropic elasticity, the Lekhnitskii formalism (Lekhnitskii 1963) and the Stroh formalism (Stroh 1958) are the two most widely used methods. The Lekhnitskii formalism begins with the stresses and can be regarded as the generalization of the Airy stress function to anisotropic elasticity. The Stroh formalism can be traced to the work of Eshelby et al. (1953) and starts with the displacements. For a two-dimensional planar problem, the applied loadings are usually considered in two different types: concentrated forces and edge dislocations. In many applications, the elastic field due to loadings applied in the interior of the material is made up of dislocations, which are defects in the material. In order to understand the motion of a dislocation in an elastic material, one needs to calculate the stress distribution induced by the dislocation. For homogeneous elastic material in an unbounded medium, the elastic field around dislocations 
or concentrated loadings is generally well known. In most engineering applications, the geometrical configurations all have finite boundaries. Thus the analysis of elastic fields for material with a finite boundary is necessary. Because of the complexity of the problem with a finite boundary, there are few analytical results available in the literature. On the other hand, the layered half-plane subjected to surface traction is applicable in a number of engineering fields. For example, the coating of a thin layer to protect soft matrices under contact and friction is a particular case of the problem. Furthermore, a wide range of electronic components are now manufactured by depositing semiconducting layers on supporting substrates. To develop our understanding of the characteristics of these devices, a detailed knowledge of the behaviour of dislocations in thin-film structures is needed. In this study, the solutions of layered half-plane media with a traction-free boundary and a perfectly bonded interface subjected to concentrated forces or edge dislocations in the interior of the material are presented. The Lekhnitskii formalism will be employed in conjunction with the Fourier-transform technique to obtain analytic explicit solutions.

Many research ventures start by addressing problems in a semi-infinite plane subjected to concentrated forces or dislocations, the simplest example of a medium with a boundary, for the first step of analysis. Head (1953) solved the problem for an isotropic half-plane. Ting \& Barnett (1993) discussed the relation between the image force on a dislocation and a half-plane with a fixed boundary for an anisotropic material. Further work, leading to a bimaterial which consists of two perfectly bound semi-infinite materials, has been done by Dundurs \& Sendeckyj (1965) for isotropic material and by Barnett \& Lothe (1974), Suo (1990) and Ting (1992) for the anisotropic case. The more advanced and complicated problem is supplied by a loading in an infinite strip or in an infinite layer bonded on a semi-infinite substrate. Buchwald (1964) showed that the solution of an isotropic infinite strip with Fourier integrals consisted of classic beam stretching and bending solutions from the singularities of the integrands. Nabarro \& Kostlan (1978) and Moss \& Hoover (1978) solved the problem of an edge dislocation in an isotropic strip with free surfaces simultaneously. A similar problem of an orthotropic strip was completed by Chou (1963). Stagni \& Lizzio (1986) obtained the elastic field of an isotropic infinite strip by means of the complex-variable method. They constructed the solution by using Muskhelishvili's (1975) complex potentials with the aid of Laurent's series expansions. Tullini \& Savoia (1999) solved the interior problem of an orthotropic strip subjected to a given continuous distribution of normal and shear loads. Wu \& Chiu (1995) and Chiu \& Wu (1998) obtained the solution of an anisotropic infinite strip subjected to dislocations and concentrated forces from eigenfunction expansion by residue theory, respectively. The stress distribution of an edge dislocation in an isotropic layered half-plane was investigated by Weeks et al. (1968) and Lee \& Dundurs (1973).

For anisotropic layered half-plane media, the problem becomes more complicated than that of their isotropic counterparts, due to the presence of many elastic constants. The goal of this study is to construct Green's function for planar anisotropic layered half-plane media subjected to concentrated forces or edge dislocations in the interior. We will show that the solution obtained from the mathematical method proposed in this study has its own physical meaning, the method of images. The method of images is a technique that uses a simple fundamental solution in an infinite plane to construct the solution for other more complicated problems.

Proc. R. Soc. Lond. A (2002) 
The use of the image method in solving two-dimensional isotropic problems dealing with screw dislocations is well known and has been used successfully for simple cases of multi-layered structures. It was found that an infinite number of image screw dislocations were required. The concept of semi-reflection and semi-transmission mirrors were used to determine the magnitudes and locations of the image screw dislocations without solving the boundary-value problem (Chou 1966; Kamat et al. 1987; Öveçoğlu et al. 1987). The multiple-image problem for screw dislocations then becomes a combinatorial problem of counting reflections and transmissions for a given image path length. Basically, one can extend this methodology to include any number of layers, but as the number of layers increases, obtaining an explicit solution becomes extremely labourious and time consuming. Hence the work was not extended beyond the five-layer case (with three finite lengths) by Kamat et al. (1987) and Öveçoğlu et al. (1987). By using the Fourier-transform technique and a series expansion, an effective analytical methodology was developed by Lin \& Ma (2000) to construct explicit analytical solutions for an anisotropic multi-layered medium with $n$ layers (the thickness in each layer is different), due to a screw dislocation in an arbitrary layer. The mathematical approach proposed by Lin \& Ma (2000) is indeed the image method that provides an automatic determination for the magnitudes and locations of all the image screw dislocations. This is the most general case for the problem of screw dislocations in multi-layered media. For the isotropic edge-dislocation case, however, the locations of the images are independent of the material property and are the same as those for isotropic screw dislocations, but the image singularities on the image point are more complicated than the anti-plane one (Ma \& Lin 2001). Due to the complexity and difficulty of the edge-dislocation problem, the solutions for a strip obtained by Stagni \& Lizzio (1986) cannot be easily represented in terms of an infinite series, but must be expressed in terms of recursion formulae for the unknown quantities. On the other hand, for an anisotropic half-plane problem, the image point is not on the opposite side of the applied point. In general, the locations of image singularities for an anisotropic half-plane are different and depend on the anisotropic elastic constants (Ting 1996). In the planar anisotropic half-plane problem, only five image points are required; four points for the in-plane problem and one point for the anti-plane problem. However, it is difficult to use the conventional image method to obtain the solution of the anisotropic layered half-plane problem directly.

In this study, an anisotropic layer perfectly bonded to an anisotropic dissimilar half-plane (as shown in figure 1) and subjected to in-plane concentrated forces and edge dislocations applied either in the thin layer or in the half-plane is investigated. The main objective is to develop an effective mathematical method to construct analytical full-field solutions for this problem. The solution procedure follows Lekhnitskii's formalism and the Fourier transform. The analytical solutions for the stresses obtained in this study are exact and are expressed in an explicit closed form. The complete solutions consist only of the simplest solutions for an infinite homogeneous medium with concentrated forces and edge dislocations. It can be shown that the physical meaning of the solution obtained in this study is the image method. The magnitudes and locations of image singularities will be determined automatically from the mathematical method presented in the study. For the special case when the thickness of the layer is infinitely large, the image singularities obtained by Ting (1992) for anisotropic bimaterials are recovered. The given formulae will

Proc. R. Soc. Lond. A (2002) 
allow a thorough assessment of the significance of anisotropy in many areas of study. Numerical results for the full-field stress distribution based on the analytical solutions are presented.

\section{Material constants and governing equations of a planar anisotropic material}

The equilibrium equations for the elastostatic problem in the absence of body forces are

$$
\sigma_{i j, j}=0,
$$

where the repeated indices imply summation and a subscript comma denotes differentiation. The generalized Hooke's law for an anisotropic, homogeneous and linearly elastic solid is given by

$$
\sigma_{i j}=C_{i j k l} \varepsilon_{k l}
$$

where $\varepsilon_{k l}$ denotes the infinitesimal strain tensor and the $C_{i j k l}$ are the elasticity constants, which are assumed to be fully symmetric and positive-definite.

Introducing the contracted notation, the stress-strain law in (2.2) can be written in a matrix form as

or

$$
\left[\begin{array}{l}
\sigma_{x x} \\
\sigma_{y y} \\
\sigma_{z z} \\
\sigma_{y z} \\
\sigma_{x z} \\
\sigma_{x y}
\end{array}\right]=\left[\begin{array}{cccccc}
C_{11} & C_{12} & C_{13} & C_{14} & C_{15} & C_{16} \\
& C_{22} & C_{23} & C_{24} & C_{25} & C_{26} \\
& & C_{33} & C_{34} & C_{35} & C_{36} \\
& & & C_{44} & C_{45} & C_{46} \\
& \text { sym. } & & & C_{55} & C_{56} \\
& & & & & C_{66}
\end{array}\right]\left[\begin{array}{l}
\varepsilon_{x x} \\
\varepsilon_{y y} \\
\varepsilon_{z z} \\
\varepsilon_{y z} \\
\varepsilon_{x z} \\
\varepsilon_{x y}
\end{array}\right]
$$

$$
\left[\begin{array}{l}
\varepsilon_{x x} \\
\varepsilon_{y y} \\
\varepsilon_{z z} \\
\varepsilon_{y z} \\
\varepsilon_{x z} \\
\varepsilon_{x y}
\end{array}\right]=\left[\begin{array}{cccccc}
a_{11} & a_{12} & a_{13} & a_{14} & a_{15} & a_{16} \\
& a_{22} & a_{23} & a_{24} & a_{25} & a_{26} \\
& & a_{33} & a_{34} & a_{35} & a_{36} \\
& & & a_{44} & a_{45} & a_{46} \\
& \text { sym. } & & & a_{55} & a_{56} \\
& & & & & a_{66}
\end{array}\right]\left[\begin{array}{c}
\sigma_{x x} \\
\sigma_{y y} \\
\sigma_{z z} \\
\sigma_{y z} \\
\sigma_{x z} \\
\sigma_{x y}
\end{array}\right],
$$

where $C_{i j}$ and $a_{i j}$ are the elastic stiffnesses and elastic compliances of an anisotropic material, respectively.

For a general anisotropic material, the in-plane and anti-plane deformations are coupled. When the compliance matrix (or stiffness matrix) of an anisotropic material possesses the form

$$
\left[a_{i j}\right]=\left[\begin{array}{cccccc}
a_{11} & a_{12} & a_{13} & 0 & 0 & a_{16} \\
& a_{22} & a_{23} & 0 & 0 & a_{26} \\
& & a_{33} & a_{34} & a_{35} & a_{36} \\
& & & a_{44} & a_{45} & 0 \\
& \text { sym. } & & & a_{55} & 0 \\
& & & & & a_{66}
\end{array}\right]
$$

then the in-plane and anti-plane deformation will be uncoupled. The problem associated with the in-plane problem is known as the planar anisotropic problem. In fact, 
monoclinic materials with the symmetry plane at $z=0$ not only satisfy the condition of (2.5), but also require $a_{34}=a_{35}=0$.

For a two-dimensional planar anisotropic problem, the stress-strain relations are only dependent on six independent material constants and can be presented in the matrix form as follows. For plane-stress problems,

$$
\left[\begin{array}{l}
\varepsilon_{x x} \\
\varepsilon_{y y} \\
\varepsilon_{x y}
\end{array}\right]=\left[\begin{array}{lll}
a_{11} & a_{12} & a_{16} \\
a_{12} & a_{22} & a_{26} \\
a_{16} & a_{26} & a_{66}
\end{array}\right]\left[\begin{array}{l}
\sigma_{x x} \\
\sigma_{y y} \\
\sigma_{x y}
\end{array}\right]
$$

and $\sigma_{z z}=\sigma_{x z}=\sigma_{y z}=0$. For plane-strain problems,

$$
\left[\begin{array}{l}
\varepsilon_{x x} \\
\varepsilon_{y y} \\
\varepsilon_{x y}
\end{array}\right]=\left[\begin{array}{lll}
s_{11} & s_{12} & s_{16} \\
s_{12} & s_{22} & s_{26} \\
s_{16} & s_{26} & s_{66}
\end{array}\right]\left[\begin{array}{l}
\sigma_{x x} \\
\sigma_{y y} \\
\sigma_{x y}
\end{array}\right]
$$

and $\varepsilon_{z z}=\varepsilon_{x z}=\varepsilon_{y z}=0$, where the $s_{i j}$ are the reduced elastic compliances. The relationship between $s_{i j}$ and $a_{i j}$ is

$$
s_{i j}=a_{i j}-\frac{a_{i 3} a_{j 3}}{a_{33}}, \quad i, j=1,2,6 .
$$

In many applications, the choice of the coordinate is very often dictated by the boundary conditions of the problem and hence may not coincide with the symmetry planes of the material. Therefore, transformation of the elastic compliances to a different coordinate system is necessary. For the special case in which the transformation is a rotation, the $z$-axis with an angle $\theta$, the elastic compliances in the new coordinate system $\left(x^{\prime}, y^{\prime}, z^{\prime}\right)$ can be presented as

$$
\boldsymbol{s}^{\prime}=\left(\boldsymbol{T}^{-1}\right)^{\mathrm{T}} \boldsymbol{s} \boldsymbol{T}^{-1},
$$

where

$$
\boldsymbol{T}=\left[\begin{array}{ccc}
\cos ^{2} \theta & \sin ^{2} \theta & \sin 2 \theta \\
\sin ^{2} \theta & \cos ^{2} \theta & -\sin 2 \theta \\
-\cos \theta \sin \theta & \cos \theta \sin \theta & \cos ^{2} \theta-\sin ^{2} \theta
\end{array}\right] .
$$

For a two-dimensional problem, the in-plane stresses can be represented by the Airy stress function $\Phi(x, y)$ as follows:

$$
\sigma_{x x}=\frac{\partial^{2} \Phi}{\partial y^{2}}, \quad \sigma_{y y}=\frac{\partial^{2} \Phi}{\partial x^{2}}, \quad \sigma_{x y}=-\frac{\partial^{2} \Phi}{\partial x \partial y} .
$$

In order to satisfy the compatibility equation, the governing equation of planar anisotropy in the absence of body forces can be obtained as

$$
s_{22} \frac{\partial^{4} \Phi}{\partial x^{4}}-2 s_{26} \frac{\partial^{4} \Phi}{\partial x^{3} \partial y}+\left(2 s_{12}+s_{66}\right) \frac{\partial^{4} \Phi}{\partial x^{2} \partial y^{2}}-2 s_{16} \frac{\partial^{4} \Phi}{\partial x \partial y^{3}}+s_{11} \frac{\partial^{4} \Phi}{\partial y^{4}}=0
$$

for plane strain and

$$
a_{22} \frac{\partial^{4} \Phi}{\partial x^{4}}-2 a_{26} \frac{\partial^{4} \Phi}{\partial x^{3} \partial y}+\left(2 a_{12}+a_{66}\right) \frac{\partial^{4} \Phi}{\partial x^{2} \partial y^{2}}-2 a_{16} \frac{\partial^{4} \Phi}{\partial x \partial y^{3}}+a_{11} \frac{\partial^{4} \Phi}{\partial y^{4}}=0
$$

for plane stress. The two governing equations are similar, and the difference between (2.11) and (2.12) is the material constants.

Proc. R. Soc. Lond. A (2002) 


\section{Green's function of an anisotropic infinite plane}

Consider an anisotropic infinite space subjected to a line of uniformly distributed forces $\boldsymbol{f}$ per unit length applied on the $z$-axis and in the direction that is normal to the $z$-axis. In addition, there is a line dislocation at the $z$-axis, with Burgers's vector $\boldsymbol{b}$ normal to the $z$-axis. This is a two-dimensional planar anisotropic problem. For the plane-strain problem, the governing equation is shown in (2.11). The expression for the field variables will be found by applying a Fourier transform over the spatial coordinate $x$ with parameter $\omega$. Take the Fourier-transform pairs of a function $g(x, y)$, defined as

$$
\tilde{g}(\omega, y)=\int_{-\infty}^{\infty} g(x, y) \mathrm{e}^{-\mathrm{i} \omega x} \mathrm{~d} x, \quad g(x, y)=\frac{1}{2 \pi} \int_{-\infty}^{\infty} \tilde{g}(\omega, y) \mathrm{e}^{\mathrm{i} \omega x} \mathrm{~d} \omega,
$$

and apply to the governing equation (2.11). Then (2.11) becomes an ordinary differential equation,

$$
s_{22} \omega^{4}+2 s_{26} \mathrm{i} \omega^{3} \frac{\mathrm{d} \tilde{\Phi}}{\mathrm{d} y}-\left(2 s_{12}+s_{66}\right) \omega^{2} \frac{\mathrm{d}^{2} \tilde{\Phi}}{\mathrm{d} y^{2}}-2 s_{16} \mathrm{i} \omega \frac{\mathrm{d}^{3} \tilde{\Phi}}{\mathrm{d} y^{3}}+s_{11} \frac{\mathrm{d}^{4} \tilde{\Phi}}{\mathrm{d} y^{4}}=0,
$$

with the general solutions

$$
\tilde{\Phi}(\omega, y)=\sum_{j=1}^{4} c_{j} \mathrm{e}^{\varsigma_{j} \omega y},
$$

where $c_{j}$ are unknown constants to be determined and $\varsigma_{j}$ are the roots of the following characteristic equation:

$$
s_{22}+2 \mathrm{i} s_{26} \varsigma-\left(2 s_{12}+s_{66}\right) \varsigma^{2}-2 \mathrm{i} s_{16} \varsigma^{3}+s_{11} \varsigma^{4}=0 .
$$

There are four roots for $\varsigma$, all of which are complex numbers. The magnitudes $\varsigma_{j}$ are related to the eigenvalues of the elastic constant $p_{j}$ of the Lekhnitskii or Stroh formalism as

$$
\varsigma_{j}=\mathrm{i} p_{j}, \quad j=1,2,3,4,
$$

where $p_{k}=\alpha_{k}+\mathrm{i} \beta_{k}, p_{k+2}=\bar{p}_{k}, k=1,2$, and $\alpha_{k}$ and $\beta_{k}$ are real, with $\beta_{k}>0$.

The general solutions for stresses and displacements of planar anisotropic material in the Fourier-transform domain are represented in a compact matrix form as

$$
\left[\begin{array}{c}
\tilde{\boldsymbol{\sigma}} \\
\tilde{\boldsymbol{u}}
\end{array}\right]=\boldsymbol{P}\left\langle\mathrm{e}^{\mathrm{i} \omega p_{*} y}\right\rangle \boldsymbol{c}
$$

where

$$
\begin{gathered}
\tilde{\boldsymbol{\sigma}}=\frac{1}{\omega^{2}}\left[\begin{array}{c}
\tilde{\sigma}_{y y} \\
\tilde{\sigma}_{x y}
\end{array}\right], \quad \tilde{\boldsymbol{u}}=\frac{1}{\mathrm{i} \omega}\left[\begin{array}{l}
\tilde{u}_{x} \\
\tilde{u}_{y}
\end{array}\right], \\
\boldsymbol{P}=\left[\begin{array}{cccc}
-1 & -1 & -1 & -1 \\
p_{1} & p_{2} & p_{3} & p_{4} \\
\xi\left(p_{1}\right) & \xi\left(p_{2}\right) & \xi\left(p_{3}\right) & \xi\left(p_{4}\right) \\
\eta\left(p_{1}\right) & \eta\left(p_{2}\right) & \eta\left(p_{3}\right) & \eta\left(p_{4}\right)
\end{array}\right], \quad \boldsymbol{c}=\left[\begin{array}{l}
c_{1} \\
c_{2} \\
c_{3} \\
c_{4}
\end{array}\right]
\end{gathered}
$$

and $\left\langle\mathrm{e}^{\mathrm{i} \omega p_{*} y}\right\rangle$ is the diagonal matrix, i.e.

$$
\left\langle\mathrm{e}^{\mathrm{i} \omega p_{*} y}\right\rangle=\operatorname{diag}\left[\mathrm{e}^{\mathrm{i} \omega p_{1} y}, \mathrm{e}^{\mathrm{i} \omega p_{2} y}, \mathrm{e}^{\mathrm{i} \omega p_{3} y}, \mathrm{e}^{\mathrm{i} \omega p_{4} y}\right],
$$

Proc. R. Soc. Lond. A (2002) 
in which $u_{x}$ and $u_{y}$ are displacement components in the $x$ - and $y$-axis, respectively, and

$$
\xi\left(p_{j}\right)=s_{11} p_{j}^{2}+s_{12}-s_{16} p_{j}, \quad \eta\left(p_{j}\right)=s_{12} p_{j}+\frac{s_{22}}{p_{j}}-s_{26} .
$$

Consider the loadings $\boldsymbol{f}$ and $\boldsymbol{b}$ which are applied at $(x, y)=(0, d)$ in an infinite plane. The jump conditions for applied forces and dislocations are

$$
\left.\left[\begin{array}{l}
\tilde{\boldsymbol{\sigma}} \\
\tilde{\boldsymbol{u}}
\end{array}\right]\right|_{y=d^{+}}-\left.\left[\begin{array}{l}
\tilde{\boldsymbol{\sigma}} \\
\tilde{\boldsymbol{u}}
\end{array}\right]\right|_{y=d^{-}}=\left[\begin{array}{c}
-\boldsymbol{f} \\
-(1 / \mathrm{i} \omega) \boldsymbol{b}
\end{array}\right]
$$

where

$$
\boldsymbol{f}=\left[\begin{array}{l}
f_{y} \\
f_{x}
\end{array}\right] \quad \text { and } \quad \boldsymbol{b}=\left[\begin{array}{l}
b_{x} \\
b_{y}
\end{array}\right] .
$$

Substituting (3.5) into (3.6), we get

$$
\boldsymbol{c}^{+}-\boldsymbol{c}^{-}=\frac{1}{\omega^{2}}\left\langle\mathrm{e}^{-\mathrm{i} \omega p_{*} d}\right\rangle \boldsymbol{q},
$$

where

$$
\boldsymbol{q}=\left[\begin{array}{l}
q_{1} \\
q_{2} \\
q_{3} \\
q_{4}
\end{array}\right]=\boldsymbol{P}^{-1}\left[\begin{array}{c}
-\boldsymbol{f} \\
\boldsymbol{b}
\end{array}\right] .
$$

The vector $\boldsymbol{q}$ can be regarded as the strength of the applied loadings for anisotropic infinite plane rather than the force $\boldsymbol{f}$ and dislocation $\boldsymbol{b}$.

After taking the inverse Fourier transform, Green's functions of stresses and displacements for the infinite plane are

$$
\left.\begin{array}{rl}
{\left[\begin{array}{l}
\sigma_{y y} \\
\sigma_{x y}
\end{array}\right]=} & \frac{1}{\pi} \operatorname{Re}\left\{\begin{array}{c}
-\frac{\mathrm{i} q_{1}}{x+p_{1}(y-d)}-\frac{\mathrm{i} q_{2}}{x+p_{2}(y-d)} \\
\frac{\mathrm{i} p_{1} q_{1}}{x+p_{1}(y-d)}+\frac{\mathrm{i} p_{2} q_{2}}{x+p_{2}(y-d)}
\end{array}\right\} \\
= & \frac{1}{\pi} \operatorname{Re}\left\{\begin{array}{l}
\sum_{j=1}^{2} \frac{-\mathrm{i} q_{j}}{x+p_{j}(y-d)} \\
\sum_{j=1}^{2} \frac{\mathrm{i} p_{j} q_{j}}{x+p_{j}(y-d)}
\end{array}\right\}, \\
{\left[\begin{array}{l}
u_{x} \\
u_{y}
\end{array}\right]=-\frac{1}{\pi} \operatorname{Re}\left\{\begin{array}{l}
\xi\left(p_{3}\right) q_{1} \ln \left(x+p_{1}(y-d)\right)+\xi\left(p_{4}\right) q_{2} \ln \left(x+p_{2}(y-d)\right) \\
\eta\left(p_{3}\right) q_{1} \ln \left(x+p_{1}(y-d)\right)+\eta\left(p_{4}\right) q_{2} \ln \left(x+p_{2}(y-d)\right)
\end{array}\right\},}
\end{array}\right\}
$$

where Re denotes the real part. The stresses display a singularity of order $1 / r$ as the distance $r$ from the applied loadings tends to zero. In the next section, we will show that Green's functions for the layered half-plane derived in this study are composed of the fundamental solutions of an infinite plane as presented in (3.8).

Proc. R. Soc. Lond. A (2002) 


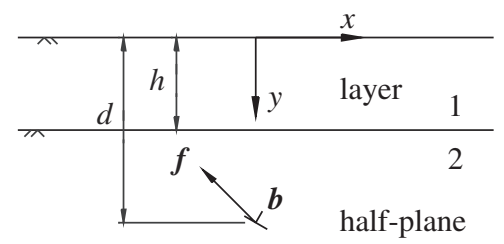

Figure 1. Configuration and coordinate system of an anisotropic layered half-plane.

\section{Green's function for an anisotropic layered half-plane}

Consider a two-dimensional problem of an anisotropic layered half-plane subjected to a concentrated force and an edge dislocation, as shown in figure 1. Assume the thin layer with thickness $h$ is occupied by material 1 and is perfectly bonded to the half-plane occupied by material 2 . The $x$-axis is taken to be the surface of the thin layer. The thin layer occupies $0<y<h$, while the underlying half-plane (the substrate) occupies $h<y<\infty$. A concentrated force $\boldsymbol{f}$ and an edge dislocation with Burgers's vector $\boldsymbol{b}$ are applied at $(x, y)=(0, d), d>0$, which may be located in the thin layer, the half-plane, the free surface or the interface of the layered half-plane. In each case, it is required to evaluate the stresses arising in both the thin layer and the half-plane. From the general solution presented in (3.5), the solutions in the Fourier-transform domain for materials 1 (thin layer) and 2 (half-plane) can be expressed as

$$
\begin{aligned}
& {\left[\begin{array}{l}
\tilde{\boldsymbol{\sigma}}^{(1)} \\
\tilde{\boldsymbol{u}}^{(1)}
\end{array}\right]=\boldsymbol{P}^{(1)}\left\langle\mathrm{e}^{\mathrm{i} \omega p_{*}^{(1)} y}\right\rangle \boldsymbol{c}^{(1)},} \\
& {\left[\begin{array}{l}
\tilde{\boldsymbol{\sigma}}^{(2)} \\
\tilde{\boldsymbol{u}}^{(2)}
\end{array}\right]=\boldsymbol{P}^{(2)}\left\langle\mathrm{e}^{\mathrm{i} \omega p_{*}^{(2)} y}\right\rangle \boldsymbol{c}^{(2)} .}
\end{aligned}
$$

The superscripts (1) and (2) on the field quantities are employed to label materials 1 and 2, respectively. The boundary conditions on the top surface of the thin layer are traction free, thus

$$
\tilde{\boldsymbol{\sigma}}^{(1)}(\omega, 0)=0 .
$$

In terms of $c_{j}^{(1)}$ from (4.1), we have

$$
\begin{aligned}
c_{1}^{(1)}+c_{2}^{(1)}+c_{3}^{(1)}+c_{4}^{(1)} & =0, \\
p_{1}^{(1)} c_{1}^{(1)}+p_{2}^{(1)} c_{2}^{(1)}+p_{3}^{(1)} c_{3}^{(1)}+p_{4}^{(1)} c_{4}^{(1)} & =0 .
\end{aligned}
$$

The continuity conditions of stresses and displacements at the interface $y=h$ are

$$
\left[\begin{array}{c}
\tilde{\boldsymbol{\sigma}}^{(1)} \\
\tilde{\boldsymbol{u}}^{(1)}
\end{array}\right]=\left[\begin{array}{c}
\tilde{\boldsymbol{\sigma}}^{(2)} \\
\tilde{\boldsymbol{u}}^{(2)}
\end{array}\right] \quad \text { at } y=h .
$$

We rewrite (4.5) in terms of $\boldsymbol{c}^{(1)}$ and $\boldsymbol{c}^{(2)}$ as

$$
\boldsymbol{P}^{(1)}\left\langle\mathrm{e}^{\mathrm{i} \omega p_{*}^{(1)} h}\right\rangle \boldsymbol{c}^{(1)}=\boldsymbol{P}^{(2)}\left\langle\mathrm{e}^{\mathrm{i} \omega p_{*}^{(2)} h}\right\rangle \boldsymbol{c}^{(2)} .
$$

The bounded solution of stresses and displacements of the half-plane requires that

$$
c_{3}^{(2)}=c_{4}^{(2)}=0 .
$$

The complete solutions for the applied loadings in the interior of thin layer and in the half-plane are different. Thus the problems are considered for two situations. 


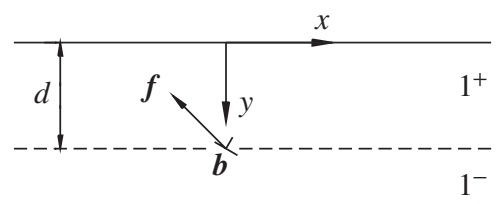

Figure 2. The jump conditions for applied loadings at the layer.

(a) The loadings are applied in the thin layer $(0 \leqslant d<h)$

When the loadings are applied in the thin layer, the jump conditions for the thin layer are employed,

$$
\left.\left[\begin{array}{c}
\tilde{\boldsymbol{\sigma}}^{(1)} \\
\tilde{\boldsymbol{u}}^{(1)}
\end{array}\right]\right|_{y=d^{+}}-\left.\left[\begin{array}{c}
\tilde{\boldsymbol{\sigma}}^{(1)} \\
\tilde{\boldsymbol{u}}^{(1)}
\end{array}\right]\right|_{y=d^{-}}=\left[\begin{array}{c}
-\boldsymbol{f} \\
-(1 / \mathrm{i} \omega) \boldsymbol{b}
\end{array}\right]
$$

The associated configuration of the jump condition is shown in figure 2 . When we express (4.8) in terms of $\boldsymbol{c}^{(1)}$, we have

$$
\left(\boldsymbol{c}^{(1)}\right)^{+}-\left(\boldsymbol{c}^{(1)}\right)^{-}=\frac{1}{\omega^{2}}\left\langle\mathrm{e}^{-\mathrm{i} \omega p_{*}^{(1)}} d\right\rangle \boldsymbol{q}^{(1)},
$$

where superscripts ' + ' and ' - ' indicate the undetermined constant $\boldsymbol{c}^{(1)}$ in (4.1) for $0 \leqslant y \leqslant d$ and $d \leqslant y \leqslant h$, respectively, and

$$
\boldsymbol{q}^{(1)}=\left(\boldsymbol{P}^{(1)}\right)^{-1}\left[\begin{array}{c}
-\boldsymbol{f} \\
\boldsymbol{b}
\end{array}\right] .
$$

With the aid of boundary conditions (4.3), (4.4) and (4.7), continuity conditions $(4.6)$ and by omitting the algebraic derivation, the undetermined constants $c_{j}^{(1)}$ and $c_{k}^{(2)}$ can be expressed explicitly and are summarized as

$$
\begin{aligned}
c_{j}^{(1)} & =\sum_{k=1}^{2} b_{j k}^{\prime} c_{k}^{(2)} \mathrm{e}^{\mathrm{i} \omega\left(p_{k}^{(2)}-p_{j}^{(1)}\right) h}, \\
c_{k}^{(2)} & =\frac{1}{\omega^{2}} \frac{(-1)^{k}}{\operatorname{det}|*|} \sum_{l=1}^{4} \sum_{m=1}^{4} p_{l m}^{-} b_{m(3-k)}^{\prime} q_{l}^{(1)} \mathrm{e}^{-\mathrm{i} \omega\left(\left(p_{m}^{(1)}+p_{k}^{(2)}\right) h+p_{l}^{(1)} d\right)},
\end{aligned}
$$

where

$$
\begin{aligned}
\operatorname{det}|*| & =\sum_{j=1}^{3} \sum_{k=j+1}^{4} p_{k j}^{-} B_{j k} \mathrm{e}^{-\mathrm{i} \omega p_{j k}^{+} h} \\
B_{j k} & =b_{j 1}^{\prime} b_{k 2}^{\prime}-b_{j 2}^{\prime} b_{k 1}^{\prime} \\
b_{i j}^{\prime} & =\sum_{k=1}^{4}\left[P_{i k}^{(1)}\right]^{-1}\left[P_{k j}^{(2)}\right] \\
p_{i j}^{ \pm} & =p_{i}^{(1)} \pm p_{j}^{(1)}
\end{aligned}
$$

Proc. R. Soc. Lond. A (2002) 
The explicit solutions of stress functions in the Fourier-transform domain for the layered half-plane are

$$
\begin{aligned}
& \tilde{\Phi}^{(1)}=\sum_{j=1}^{4} c_{j}^{(1)} \mathrm{e}^{\mathrm{i} p_{j}^{(1)} \omega y}, \\
& \tilde{\Phi}^{(2)}=\sum_{k=1}^{2} c_{k}^{(2)} \mathrm{e}^{\mathrm{i} p_{k}^{(2)} \omega y} .
\end{aligned}
$$

Because the denominators in (4.10) and (4.11) are so complicated, it is not possible to obtain the inverse Fourier transform directly. In order to obtain the explicit analytical full-field solutions for the inverse Fourier transform, the denominator $\operatorname{det}|*|$ in $c_{j}^{(1)}$ and $c_{k}^{(2)}$ should be reformulated. By examining the structure of $\operatorname{det}|*|$, the term with magnitude $p_{21}^{-} B_{12} \mathrm{e}^{-\mathrm{i} p_{12}^{+} \omega h}$ is taken out from $\operatorname{det}|*|$, so that

$$
\frac{1}{\operatorname{det}|*|}=\frac{1}{p_{21}^{-} B_{12} \mathrm{e}^{-\mathrm{i} p_{12}^{+} \omega h}(1-\operatorname{det}|\cdot|)},
$$

where

$$
\operatorname{det}|\cdot|=1-\frac{\operatorname{det}|*|}{p_{21}^{-} B_{12} \mathrm{e}^{-\mathrm{i} p_{12}^{+} \omega h}} .
$$

It can be shown that $\operatorname{det}|\cdot|<1$ for $\omega>0$. By the expansion of (4.14) into a power series of $\operatorname{det}|\cdot|$, we obtain

$$
\frac{1}{p_{21}^{-} B_{12} \mathrm{e}^{-\mathrm{i} p_{12}^{+} \omega h}(1-\operatorname{det}|\cdot|)}=\frac{\mathrm{e}^{\mathrm{i} p_{12}^{+} \omega h}}{p_{21}^{-} B_{12}} \sum_{n=0}^{\infty}(\operatorname{det}|\cdot|)^{n} .
$$

Because only five terms are included in $\operatorname{det}|\cdot|, \sum_{n=0}^{\infty}(\operatorname{det}|\cdot|)^{n}$ can be expressed explicitly as

$$
\frac{\mathrm{e}^{\mathrm{i} p_{12}^{+} \omega h}}{p_{21}^{-} B_{12}} \sum_{n=0}^{\infty}(\operatorname{det}|\cdot|)^{n}=\sum M \mathrm{e}^{-\mathrm{i} \omega g h}
$$

where

$$
\begin{aligned}
M & =\frac{v}{\left(p_{21}^{-} B_{12}\right)^{n+1}}\left(p_{13}^{-} B_{13}\right)^{n_{1}}\left(p_{23}^{-} B_{23}\right)^{n_{2}}\left(p_{14}^{-} B_{14}\right)^{n_{3}}\left(p_{24}^{-} B_{24}\right)^{n_{4}}\left(p_{34}^{-} B_{34}\right)^{n_{5}} \\
g & =n_{1} p_{13}^{+}+n_{2} p_{23}^{+}+n_{3} p_{14}^{+}+n_{4} p_{24}^{+}+n_{5} p_{34}^{+}-(n+1) p_{12}^{+} \\
\sum & =\sum_{n=0}^{\infty} \sum_{n_{1}=0}^{n} \sum_{n_{2}=0}^{n-n_{1}} \sum_{n_{3}=0}^{n-n_{1}-n_{2}} \sum_{n_{4}=0}^{n-n_{1}-n_{2}-n_{3}} \\
n_{5} & =n-n_{1}-n_{2}-n_{3}-n_{4} \\
v & =\frac{n !}{n_{1} ! n_{2} ! n_{3} ! n_{4} ! n_{5} !}
\end{aligned}
$$

and '!' denotes factorial. By omitting the details of the derivation, the solutions for the stress functions in (4.12) and (4.13) can be expressed in the explicit forms as 
follows:

$$
\begin{aligned}
& \tilde{\Phi}^{(1)}=\frac{1}{\omega^{2}} \sum \sum_{j=1}^{4} \sum_{k=1}^{2} \sum_{l=1}^{4} \sum_{m=1}^{4}(-1)^{k} b_{j k}^{\prime} b_{m(3-k)}^{\prime} p_{l m}^{-} q_{l}^{(1)} M \mathrm{e}^{\mathrm{i} \omega\left(p_{j}^{(1)} y-\left(p_{j}^{(1)}+p_{m}^{(1)}+g\right) h-p_{l}^{(1)} d\right),} \\
& \tilde{\Phi}^{(2)}=\frac{1}{\omega^{2}} \sum \sum_{k=1}^{2} \sum_{l=1}^{4} \sum_{m=1}^{4}(-1)^{k} b_{m(3-k)}^{\prime} p_{l m}^{-} q_{l}^{(1)} M \mathrm{e}^{\mathrm{i} \omega\left(p_{k}^{(2)} y-\left(p_{m}^{(1)}+p_{k}^{(2)}+g\right) h-p_{l}^{(1)} d\right)} .
\end{aligned}
$$

Now, the solutions for $\tilde{\Phi}^{(1)}$ and $\tilde{\Phi}^{(2)}$ are linear combinations of exponential functions, i.e. $\mathcal{M} \mathrm{e}^{\mathrm{i} \omega G}$; each term represents Green's function in the transform domain for concentrated loadings in an infinite homogeneous medium. The term with $G$ in the exponential functions indicates the location of the loading, and $\mathcal{M}$ represents the magnitude of the loading. The location of the loading depends on the thickness of the thin layer $h$, the position of applied loadings $d$ and eigenvalues $p_{j}$. The magnitude of $\mathcal{M}$ only depends on material constants. Thus four terms in (4.21) are fundamental solutions of an infinite plane subjected to concentrated forces and edge dislocations. All the remaining terms in (4.21) and all the terms in (4.22) are image singularities that are induced to satisfy the boundary and interface continuity conditions. Hence this method is referred to as the generalization method of images.

Since the solutions in the transformed domain expressed in (4.21) and (4.22) are exponential functions of $\omega$, only the following inverse Fourier transformation is required,

$$
\frac{1}{2 \pi} \int_{-\infty}^{\infty} \mathrm{e}^{\mathrm{i} \omega(p y+H)} \mathrm{e}^{\mathrm{i} \omega x} \mathrm{~d} \omega=\frac{1}{\pi} \operatorname{Re}\left[\frac{\mathrm{i}}{x+p y+H}\right],
$$

where $H$ is an arbitrary constant. Therefore, the inverse Fourier transformations for (4.21) and (4.22) can easily be obtained term by term. Finally, the explicit closedform solutions of stresses for the layered half-plane are presented as follows,

$$
\left[\begin{array}{c}
\sigma_{y y}^{(j)} \\
\sigma_{x y}^{(j)} \\
\sigma_{x x}^{(j)}
\end{array}\right]=\left[\begin{array}{c}
\left.\psi^{(j)}\right|_{\alpha=0} \\
\left.\psi^{(j)}\right|_{\alpha=1} \\
\left.\psi^{(j)}\right|_{\alpha=2}
\end{array}\right], \quad j=1,2,
$$

where

$$
\begin{aligned}
\psi^{(1)}(x, y)= & \frac{1}{\pi} \sum_{n=0}^{\infty} \sum_{n_{1}=0}^{n} \sum_{n_{2}=0}^{n-n_{1}} \sum_{n_{3}=0}^{n-n_{1}-n_{2}} \sum_{n_{4}=0}^{n-n_{1}-n_{2}-n_{3}} \\
& \sum_{j=1}^{4} \sum_{l=1}^{4} \sum_{m=1}^{4} \operatorname{Re}\left[\frac{\mathrm{i}(-1)^{\alpha} B_{j m} p_{l m}^{-} q_{l}^{(1)} M\left(p_{j}^{(1)}\right)^{\alpha}}{x+p_{j}^{(1)} y-\left(p_{j m}^{+}+g\right) h-p_{l}^{(1)} d}\right] \\
= & \frac{1}{\pi} \sum_{j=1}^{2} \operatorname{Re}\left[\frac{\mathrm{i}(-1)^{\alpha+1} q_{j}^{(1)}\left(p_{j}^{(1)}\right)^{\alpha}}{x+p_{j}^{(1)}(y-d)}\right] \\
& +\frac{1}{\pi} \sum_{j=1}^{2} \sum_{l=3}^{4} \operatorname{Re}\left[\frac{p_{l(3-j)}^{-}}{p_{21}^{-}} \frac{\mathrm{i}(-1)^{j+\alpha} q_{l}^{(1)}\left(p_{j}^{(1)}\right)^{\alpha}}{x+\left(p_{j}^{(1)} y-p_{l}^{(1)} d\right)}\right]
\end{aligned}
$$

Proc. R. Soc. Lond. A (2002) 


$$
\begin{aligned}
& +\frac{1}{\pi} \sum_{j=1}^{4} \sum_{l=1}^{4} \sum_{m=1}^{4}\left(1-\delta_{(j+m) 3}\right) \operatorname{Re}\left[\frac{\mathrm{i}(-1)^{\alpha} B_{j m} p_{l m}^{-} q_{l}^{(1)} M\left(p_{j}^{(1)}\right)^{\alpha}}{x+p_{j}^{(1)} y-\left(p_{j m}^{+}+g\right) h-p_{l}^{(1)} d}\right] \\
& +\frac{1}{\pi} \sum^{\prime} \sum_{j=1}^{4} \sum_{l=1}^{4} \sum_{m=1}^{4} \operatorname{Re}\left[\frac{\mathrm{i}(-1)^{\alpha} B_{j m} p_{l m}^{-} q_{l}^{(1)} M\left(p_{j}^{(1)}\right)^{\alpha}}{x+p_{j}^{(1)} y-\left(p_{j m}^{+}+g\right) h-p_{l}^{(1)} d}\right]
\end{aligned}
$$

and

$$
\begin{aligned}
\psi^{(2)}(x, y)=\frac{1}{\pi} \sum_{n=0}^{\infty} \sum_{n_{1}=0}^{n} \sum_{n_{2}=0}^{n-n_{1}} \sum_{n_{3}=0}^{n-n_{1}-n_{2}} \sum_{n_{4}=0}^{n-n_{1}-n_{2}-n_{3}} \\
\sum_{k=1}^{2} \sum_{l=1}^{4} \sum_{m=1}^{4} \operatorname{Re}\left[\frac{\mathrm{i}(-1)^{k+\alpha} b_{m(3-k)}^{\prime} p_{m l}^{-} q_{l}^{(1)} M\left(p_{k}^{(2)}\right)^{\alpha}}{x+p_{k}^{(2)}(y-h)-\left(p_{m}^{(1)}+g\right) h-p_{l}^{(1)} d}\right] \\
=\frac{1}{\pi} \sum_{k=1}^{2} \sum_{m=1}^{2} \operatorname{Re}\left[\frac{1}{B_{12}} \frac{\mathrm{i}(-1)^{k+\alpha+m} b_{m(3-k)}^{\prime} q_{3-m}^{(1)}\left(p_{k}^{(2)}\right)^{\alpha}}{x+p_{k}^{(2)}(y-h)-\left(p_{m}^{(1)}-p_{12}^{+}\right) h-p_{3-m}^{(1)} d}\right] \\
+\frac{1}{\pi} \sum_{k=1}^{2} \sum_{l=1}^{4} \sum_{m=1}^{4}\left(1-\delta_{(l+m) 3}\right) \\
\quad \times \operatorname{Re}\left[\frac{\mathrm{i}(-1)^{k+\alpha} b_{m(3-k)}^{\prime} p_{m l}^{-} q_{l}^{(1)} M\left(p_{k}^{(2)}\right)^{\alpha}}{x+p_{k}^{(2)}(y-h)-\left(p_{m}^{(1)}+g\right) h-p_{l}^{(1)} d}\right] \\
+\frac{1}{\pi} \sum^{\prime} \sum_{k=1}^{2} \sum_{l=1}^{4} \sum_{m=1}^{4} \operatorname{Re}\left[\frac{\mathrm{i}(-1)^{k+\alpha} b_{m(3-k)}^{\prime} p_{m l}^{-} q_{l}^{(1)} M\left(p_{k}^{(2)}\right)^{\alpha}}{x+p_{k}^{(2)}(y-h)-\left(p_{m}^{(1)}+g\right) h-p_{l}^{(1)} d}\right]
\end{aligned}
$$

in which

$$
\Sigma^{\prime}=\sum_{n=1}^{\infty} \sum_{n_{1}=0}^{n} \sum_{n_{2}=0}^{n-n_{1}} \sum_{n_{3}=0}^{n-n_{1}-n_{2}} \sum_{n_{4}=0}^{n-n_{1}-n_{2}-n_{3}}
$$

and $\delta_{j k}$ is Kronecker's delta. It is noted that the whole-field solutions of stresses in the layered half-plane $\left(\psi^{(1)}(x, y)\right.$ for the thin layer and $\psi^{(2)}(x, y)$ for the half-plane) can be explicitly expressed with compact forms, which are presented in $(4.24 a)$ and $(4.25 a)$. There are eight summation operators in $\psi^{(1)}$ and $\psi^{(2)}$, but only one summation operator has infinite summation numbers, i.e. $\sum_{n=0}^{\infty}$ in $\psi^{(1)}$ and $\psi^{(2)}$. The infinite summation operator controls the convergence of the solution. Therefore, it is necessary to add all the terms in the infinite summation operator for the numerical calculation.

In order to explain the physical meaning of the terms in $(4.24 a)$, the terms with $n=0$ in the solution are taken out and are expressed in $(4.24 b)-(4.24 d)$. If the thickness of the layer is infinitely large $(h \rightarrow \infty)$, the layered half-plane solution will reduce to the half-plane solution, all the terms in $(4.24 d)$ and $(4.24 e)$ will be zero and only the terms in $(4.24 b)$ and $(4.24 c)$ will be left. The term in $(4.24 b)$ with one summation is the same as that presented in (3.8) and is the solution for an infinite 
plane with a concentrated force $\boldsymbol{f}$ and an edge dislocation $\boldsymbol{b}$ applied at the location $(0, d)$. The terms in $(4.24 c)$ with two summations are the image singularities that satisfy the free-surface condition of the top surface. The locations of these image singularities are determined by solving the equation $x+p_{j}^{(1)} y-p_{l}^{(1)} d=0(j=1,2$ and $l=3,4)$ for $x$ and $y$. There are four image singularities, which are located outside the layered half-plane and the results are identical to those obtained for the halfplane problem by Ting (1992). The image singularity at each image point consists of a concentrated force and an edge dislocation. It can be verified that all the terms in $(4.24 d)$ and $(4.24 e)$ are the multiple images of the free surface and interface of the layered half-plane. The locations of all the image singularities can be determined by solving the equation $x+p_{j}^{(1)} y-\left(p_{j m}^{+}+g\right) h-p_{l}^{(1)} d=0$ and are dependent on $d$, $h$ and the material properties of the thin layer (i.e. material 1 ).

Similarly, the complete solutions for the half-plane are presented in $(4.25 a)$, and are rewritten in an alternative form, as presented in $(4.25 b)-(4.25 d)$ by taking $n=0$ out of $(4.25 a)$. The terms in $(4.25 b)$ represent the solutions for the bimaterials that consist of two anisotropic half-planes bonded together along the interface. The solution in $(4.25 b)$ consists of the first four image singularities related to the interface. The locations of these image singularities can be determined by setting the denominator of $(4.25 b)$ equal to zero; the results are dependent on $d, h$ and the material properties for both materials.

\section{(b) The loadings are applied in the half-plane $(h \leqslant d<\infty)$}

The general solutions in the Fourier-transform domain for the loadings applied in the half-plane are the same as those presented in (4.1) and (4.2) for materials 1 and 2 , respectively. If the applied loadings are located in material 2 , then the jump conditions are

$$
\left.\left[\begin{array}{c}
\tilde{\boldsymbol{\sigma}}^{(2)} \\
\tilde{\boldsymbol{u}}^{(2)}
\end{array}\right]\right|_{y=d^{+}}-\left.\left[\begin{array}{c}
\tilde{\boldsymbol{\sigma}}^{(2)} \\
\tilde{\boldsymbol{u}}^{(2)}
\end{array}\right]\right|_{y=d^{-}}=\left[\begin{array}{c}
-\boldsymbol{f} \\
-(1 / \mathrm{i} \omega) \boldsymbol{b}
\end{array}\right]
$$

In terms of the unknown constants $\boldsymbol{c}^{(2)}$, we have

$$
\left(\boldsymbol{c}^{(2)}\right)^{+}-\left(\boldsymbol{c}^{(2)}\right)^{-}=\frac{1}{\omega^{2}}\left\langle\mathrm{e}^{-\mathrm{i} \omega p_{*}^{(2)} d}\right\rangle \boldsymbol{q}^{(2)},
$$

where

$$
\boldsymbol{q}^{(2)}=\left(\boldsymbol{P}^{(2)}\right)^{-1}\left[\begin{array}{c}
-\boldsymbol{f} \\
\boldsymbol{b}
\end{array}\right]
$$

With the boundary conditions expressed in (4.3) and (4.4), and the continuity condition in (4.6), constants $c_{j}^{(1)}$ and $c_{k}^{(2)}$ are determined and are presented in a compact form as

$$
\begin{aligned}
c_{j}^{(1)}=\frac{-1}{\omega^{2}} \sum_{k=3}^{4} b_{j k}^{\prime} q_{k}^{(2)} \mathrm{e}^{\mathrm{i} \omega\left(\left(p_{k}^{(2)}-p_{j}^{(1)}\right) h-p_{k}^{(2)} d\right)} & \\
& +\frac{1}{\omega^{2} \operatorname{det}|*|} \sum_{k=1}^{2} \sum_{l=3}^{4} \sum_{m=1}^{3} \sum_{r=m+1}^{4}(-1)^{l+s+t} b_{j k}^{\prime} p_{s t}^{-} B_{m r}^{k l} q_{l}^{(2)} \\
& \times \mathrm{e}^{\mathrm{i} \omega\left(\left(p_{l}^{(2)}-p_{j}^{(1)}+p_{m r}^{+}\right) h-p_{l}^{(2)} d\right),},
\end{aligned}
$$

Proc. R. Soc. Lond. A (2002) 


$$
\begin{aligned}
c_{k}^{(2)}= & \frac{1}{\omega^{2}} q_{k}^{(2)} \mathrm{e}^{-\mathrm{i} \omega p_{k}^{(2)}} \\
& +\frac{1}{\omega^{2} \operatorname{det}|*|} \sum_{l=3}^{4} \sum_{m=1}^{3} \sum_{r=m+1}^{4}(-1)^{l+s+t} p_{s t}^{-} B_{m r}^{k l} q_{l}^{(2)} \mathrm{e}^{\mathrm{i} \omega\left(\left(p_{l}^{(2)}+p_{m r}^{+}\right) h-p_{l}^{(2)} d\right)},
\end{aligned}
$$

where

$$
\begin{aligned}
\operatorname{det}|*| & =\sum_{m=1}^{3} \sum_{r=m+1}^{4}(-1)^{s+t} p_{s t}^{-} B_{m r}^{33} \mathrm{e}^{\mathrm{i} \omega p_{m r}^{+} h}, \\
s & =3-m+\frac{1}{2}(3-r)(4-r)+\frac{1}{2}(m-2)(m-1), \\
t & =10-m-r-s, \\
b_{i j}^{\prime \prime} & =\sum_{k=1}^{4}\left[P_{i k}^{(2)}\right]^{-1}\left[P_{k j}^{(1)}\right] \\
B_{m r}^{k l} & =b_{k m}^{\prime \prime} b_{(7-l) r}^{\prime \prime}-b_{k r}^{\prime \prime} b_{(7-l) m}^{\prime \prime}, \\
p_{i j}^{ \pm} & =p_{i}^{(1)} \pm p_{j}^{(1)}
\end{aligned}
$$

The solutions of stress functions in the transformed domain are

$$
\begin{aligned}
& \tilde{\Phi}^{(1)}=\sum_{j=1}^{4} c_{j}^{(1)} \mathrm{e}^{\mathrm{i} p_{j}^{(1)} \omega y}, \\
& \tilde{\Phi}^{(2)}=\sum_{k=1}^{2} c_{k}^{(2)} \mathrm{e}^{\mathrm{i} p_{k}^{(2)} \omega y} .
\end{aligned}
$$

By using the method as discussed in $\S 4 a$, the denominator det $|*|$ in (4.28) and (4.29) can be reformulated by the series expansion as

$$
\begin{aligned}
\frac{1}{\operatorname{det}|*|} & =\frac{1}{p_{21}^{-} B_{33}^{33} \mathrm{e}^{\mathrm{i} \omega p_{34}^{+} h}(1-\operatorname{det}|\cdot|)} \\
& =\frac{\mathrm{e}^{-\mathrm{i} \omega p_{34}^{+} h}}{p_{21}^{-} B_{34}^{33}} \sum_{n=0}^{\infty}(\operatorname{det}|\cdot|)^{n} \\
& =\sum M \mathrm{e}^{\mathrm{i} \omega g h},
\end{aligned}
$$

where

$$
\begin{aligned}
M & =\frac{v}{\left(p_{21}^{-} B_{34}^{33}\right)^{n+1}}\left(p_{31}^{-} B_{24}^{33}\right)^{n_{1}}\left(p_{23}^{-} B_{14}^{33}\right)^{n_{2}}\left(p_{34}^{-} B_{12}^{33}\right)^{n_{3}}\left(p_{14}^{-} B_{23}^{33}\right)^{n_{4}}\left(p_{42}^{-} B_{13}^{33}\right)^{n_{5}}, \\
g & =n_{1} p_{24}^{+}+n_{2} p_{14}^{+}+n_{3} p_{12}^{+}+n_{4} p_{23}^{+}+n_{5} p_{13}^{+}-(n+1) p_{34}^{+} \\
\sum & =\sum_{n=0}^{\infty} \sum_{n_{1}=0}^{n} \sum_{n_{2}=0}^{n-n_{1}} \sum_{n_{3}=0}^{n-n_{1}-n_{2}} \sum_{n_{4}=0}^{n-n_{1}-n_{2}-n_{3}} \\
n_{5} & =n-n_{1}-n_{2}-n_{3}-n_{4} \\
v & =\frac{n !}{n_{1} ! n_{2} ! n_{3} ! n_{4} ! n_{5} !}
\end{aligned}
$$

Proc. R. Soc. Lond. A (2002) 


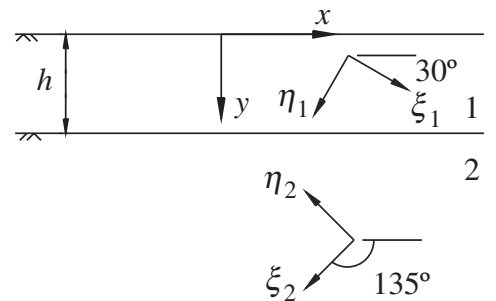

Figure 3. The configuration for rotating the principal axis of orthotropic materials 1 and 2 .

The closed-form solutions of stresses in the layered half-plane are expressed explicitly as follows,

$$
\left[\begin{array}{c}
\sigma_{y y}^{(j)} \\
\sigma_{x y}^{(j)} \\
\sigma_{x x}^{(j)}
\end{array}\right]=\left[\begin{array}{l}
\left.\psi^{(j)}\right|_{\alpha=0} \\
\left.\psi^{(j)}\right|_{\alpha=1} \\
\left.\psi^{(j)}\right|_{\alpha=2}
\end{array}\right], \quad j=1,2,
$$

with

$$
\begin{aligned}
& \psi^{(1)}(x, y) \\
& =\frac{1}{\pi} \sum_{j=1}^{4} \sum_{l=3}^{4} \operatorname{Re}\left[\frac{\mathrm{i}(-1)^{\alpha} b_{j l}^{\prime} q_{l}^{(2)}\left(p_{j}^{(1)}\right)^{\alpha}}{x+p_{j}^{(1)}(y-h)+p_{l}^{(2)}(h-d)}\right] \\
& +\frac{1}{\pi} \sum_{n=0}^{\infty} \sum_{n_{1}=0}^{n} \sum_{n_{2}=0}^{n-n_{1}} \sum_{n_{3}=0}^{n-n_{1}-n_{2}} \sum_{n_{4}=0}^{n-n_{1}-n_{2}-n_{3}} \sum_{j=1}^{4} \sum_{k=1}^{2} \sum_{l=3}^{4} \sum_{m=1}^{3} \sum_{r=m+1}^{4} \\
& \operatorname{Re}\left[\frac{\mathrm{i}(-1)^{l+s+t+\alpha} b_{j k}^{\prime} p_{t s}^{-} B_{m r}^{k l} M q_{l}^{(2)}\left(p_{j}^{(1)}\right)^{\alpha}}{x+p_{j}^{(1)}(y-h)+\left(p_{m r}^{+}+g\right) h+p_{l}^{(2)}(h-d)}\right] \\
& =\frac{1}{\pi} \sum_{j=3}^{4} \sum_{l=3}^{4} \operatorname{Re}\left[\left(b_{j l}^{\prime}-(-1)^{l} \frac{b_{j 1}^{\prime} B_{34}^{1 l}+b_{j 2}^{\prime} B_{34}^{2 l}}{B_{33}^{33}}\right) \frac{\mathrm{i}(-1)^{\alpha} q_{l}^{(2)}\left(p_{j}^{(1)}\right)^{\alpha}}{x+p_{j}^{(1)}(y-h)+p_{l}^{(2)}(h-d)}\right] \\
& +\frac{1}{\pi} \sum_{j=1}^{2} \sum_{l=3}^{4} \operatorname{Re}\left[\frac{\mathrm{i}(-1)^{\alpha} b_{j l}^{\prime} q_{l}^{(2)}\left(p_{j}^{(1)}\right)^{\alpha}}{x+p_{j}^{(1)}(y-h)+p_{l}^{(2)}(h-d)}\right] \\
& +\frac{1}{\pi} \sum_{j=1}^{4} \sum_{k=1}^{2} \sum_{l=3}^{4} \sum_{m=1}^{3} \sum_{r=m+1}^{\lambda} \operatorname{Re}\left[\frac{\mathrm{i}(-1)^{l+s+t+\alpha} b_{j k}^{\prime} p_{t s}^{-} B_{m r}^{k l} M q_{l}^{(2)}\left(p_{j}^{(1)}\right)^{\alpha}}{x+p_{j}^{(1)}(y-h)+\left(p_{m r}^{+}+g\right) h+p_{l}^{(2)}(h-d)}\right] \\
& +\frac{1}{\pi} \sum^{\prime} \sum_{j=1}^{4} \sum_{k=1}^{2} \sum_{l=3}^{4} \sum_{m=1}^{3} \sum_{r=m+1}^{4} \\
& \operatorname{Re}\left[\frac{\mathrm{i}(-1)^{l+s+t+\alpha} b_{j k}^{\prime} p_{t s}^{-} B_{m r}^{k l} M q_{l}^{(2)}\left(p_{j}^{(1)}\right)^{\alpha}}{x+p_{j}^{(1)}(y-h)+\left(p_{m r}^{+}+g\right) h+p_{l}^{(2)}(h-d)}\right],
\end{aligned}
$$

Proc. R. Soc. Lond. A (2002) 
where

$$
\lambda= \begin{cases}3, & j=3,4 \\ 4, & j=1,2\end{cases}
$$

and

$$
\begin{aligned}
& \psi^{(2)}(x, y)=\frac{1}{\pi} \sum_{k=1}^{2} \operatorname{Re}\left[\frac{\mathrm{i}(-1)^{\alpha+1} q_{k}^{(2)}\left(p_{k}^{(2)}\right)^{\alpha}}{x+p_{k}^{(2)}(y-d)}\right] \\
&+\frac{1}{\pi} \sum_{n=0}^{\infty} \sum_{n_{1}=0}^{n} \sum_{n_{2}=0}^{n-n_{1}} \sum_{n_{3}=0}^{n-n_{1}-n_{2}} \sum_{n_{4}=0}^{n-n_{1}-n_{2}-n_{3}} \sum_{k=1}^{2} \sum_{l=3}^{4} \sum_{m=1}^{3} \sum_{r=m+1}^{4} \\
& \operatorname{Re}\left[\frac{\mathrm{i}(-1)^{l+s+t+\alpha} p_{t s}^{-} B_{m r}^{k l} M q_{l}^{(2)}\left(p_{k}^{(2)}\right)^{\alpha}}{x+p_{k}^{(2)}(y-h)+\left(p_{m r}^{+}+g\right) h+p_{l}^{(2)}(h-d)}\right] .
\end{aligned}
$$

The terms in $(4.34 b)$ represent the four image singularities related to the interface. The terms in (4.35) with one summation represent Green's function of material 2 in an infinite plane with a concentrated force $\boldsymbol{f}$ and an edge dislocation $\boldsymbol{b}$ applied at the location $(0, d)$.

The analytical solutions of stresses for loadings applied either in the layer or in the half-plane can be expressed in compact forms. Each term in the complete solution represents Green's functions of simplest solutions for concentrated forces and edge dislocations in an infinite plane. To conclude, the physical meaning of the solutions is, indeed, the image method. The mathematical derivation in this study provides an automatic determination for the magnitude and locations of all the image singularities.

If the loadings are applied on the free surface $(d=0)$ of material 1 or at the interface $(d=h)$, the solutions can be obtained directly from that derived in $\S 4 a$ or $\S 4 b$. When the loadings are applied on the free surface of material 1 , the complete solutions can be obtained from $(4.24 a)$ and $(4.25 a)$ by setting $d=0$. However, if the loadings are applied at the interface, the solutions can be constructed from $(4.34 a)$ and (4.35) by setting $d=h$.

\section{Numerical results for stresses}

In this section, the full-field stress distributions of a layered half-plane subjected to concentrated forces or edge dislocations applied at different locations are investigated. The material of T300/934 graphite/epoxy is employed for numerical calculations. The engineering constants of the material in its principal coordinate are (Gibson 1994)

$$
E_{11}=131 \mathrm{GPa}, \quad E_{22}=10.3 \mathrm{GPa}, \quad G_{12}=6.9 \mathrm{GPa}, \quad \nu_{12}=0.22,
$$

where $E_{11}$ and $E_{22}$ are Young's moduli, $G_{12}$ is the shear modulus and $v_{12}$ is Poisson's ratio. The corresponding elastic compliance constants are

$$
\left[\begin{array}{lll}
s_{11} & s_{12} & s_{16} \\
s_{12} & s_{22} & s_{26} \\
s_{16} & s_{26} & s_{66}
\end{array}\right]=\left[\begin{array}{ccc}
7.63 & -1.68 & 0 \\
-1.68 & 97.1 & 0 \\
0 & 0 & 144.5
\end{array}\right] \times 10^{-3} \quad\left(\mathrm{GPa}^{-1}\right) .
$$

Proc. R. Soc. Lond. A (2002) 


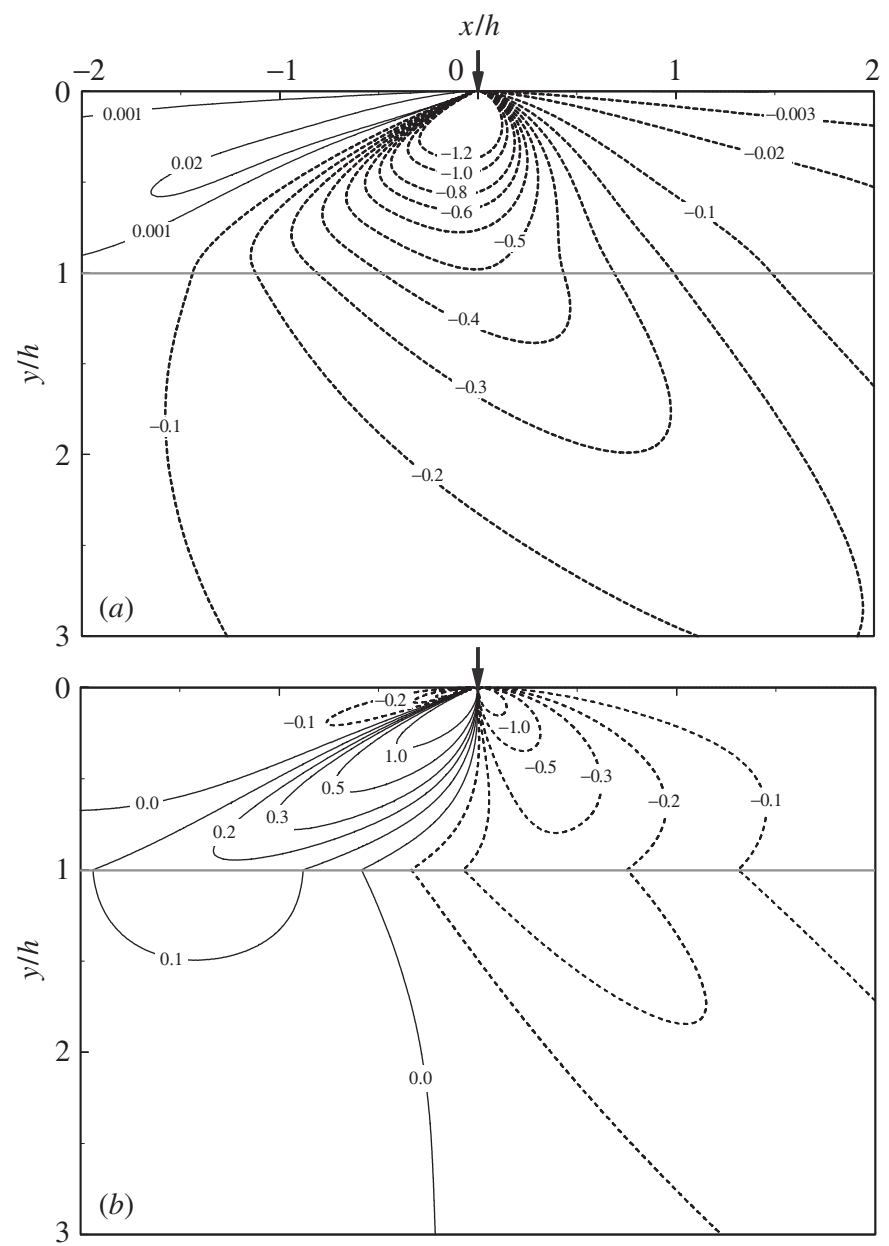

Figure 4. (a) Full-field distribution of $\sigma_{y y}$ for the layered half-plane subjected to a surface concentrated vertical force $f_{y}\left(\sigma_{y y} h / f_{y}\right)$. (b) Full-field distribution of $\sigma_{x y}$ for the layered half-plane subjected to a surface concentrated vertical force $f_{y}\left(\sigma_{x y} h / f_{y}\right)$.

In order to model the planar anisotropic of the material, the principal axis of thin layer and half-plane are rotated $\frac{1}{6} \pi$ and $\frac{3}{4} \pi$, respectively, with respect to the $x, y$ coordinate of the layered half-plane. The associated geometrical configuration is shown in figure 3 , where $\left(\xi_{1}, \eta_{1}\right)$ and $\left(\xi_{2}, \eta_{2}\right)$ indicate the principal axis of materials 1 and 2 , respectively. The elastic compliance constants of materials 1 and 2 can be obtained by using (2.9) and are expressed as follows,

$$
\left[\begin{array}{lll}
s_{11} & s_{12} & s_{16} \\
s_{12} & s_{22} & s_{26} \\
s_{16} & s_{26} & s_{66}
\end{array}\right]=\left[\begin{array}{ccc}
36.91 & -8.59 & 46.71 \\
-8.59 & 81.63 & 30.76 \\
46.71 & 30.76 & 117.3
\end{array}\right] \times 10^{-3} \quad\left(\mathrm{GPa}^{-1}\right)
$$

for material 1 , and

$$
\left[\begin{array}{lll}
s_{11} & s_{12} & s_{16} \\
s_{12} & s_{22} & s_{26} \\
s_{16} & s_{26} & s_{66}
\end{array}\right]=\left[\begin{array}{ccc}
61.57 & -10.89 & -44.73 \\
-10.89 & 61.57 & -44.73 \\
-44.73 & -44.73 & 108.1
\end{array}\right] \times 10^{-3}\left(\mathrm{GPa}^{-1}\right)
$$

Proc. R. Soc. Lond. A (2002) 

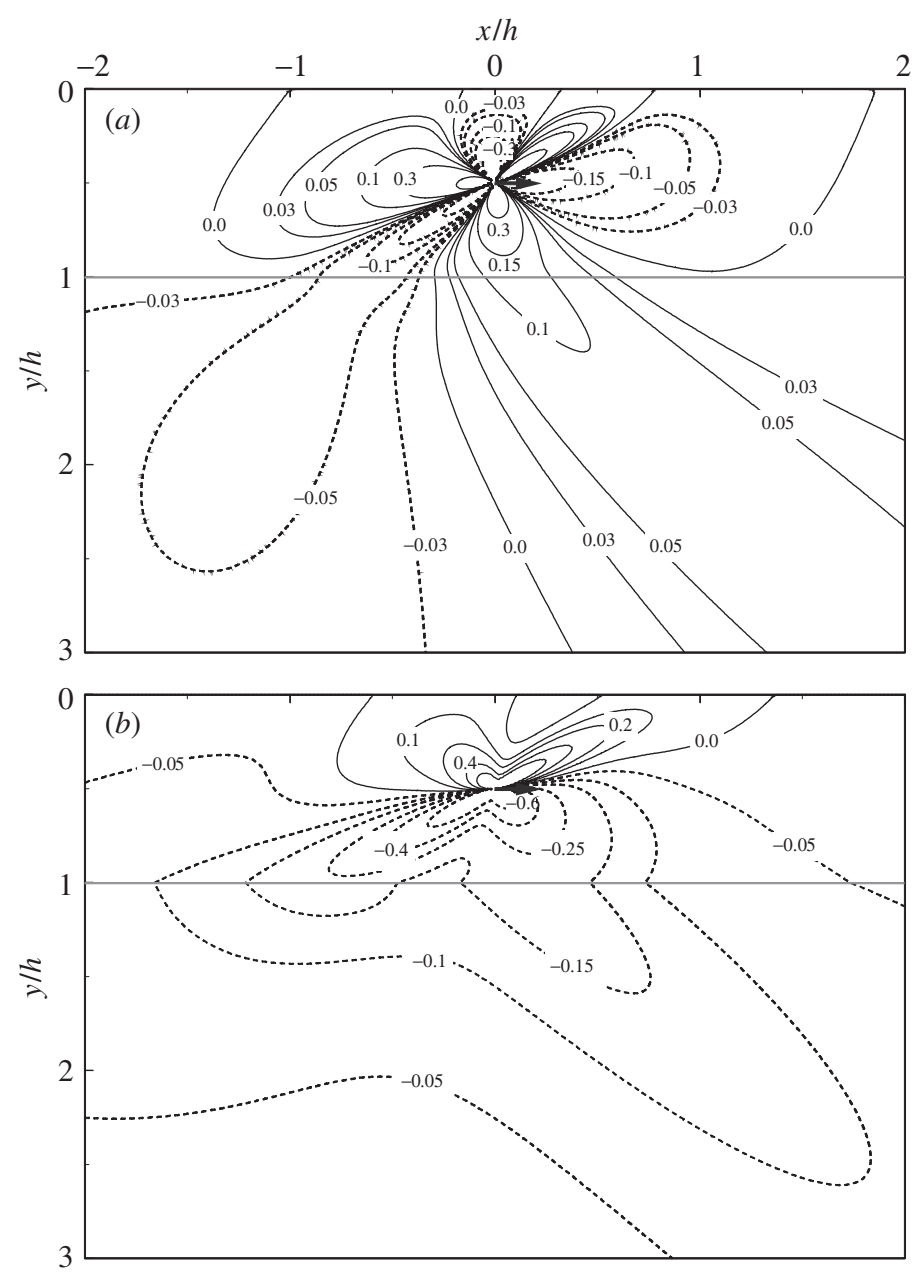

Figure 5. (a) Full-field distribution of $\sigma_{y y}$ for the layered half-plane subjected to a concentrated horizontal force $f_{x}$ in the layer $\left(\sigma_{y y} h / f_{x}\right)$. (b) Full-field distribution of $\sigma_{x y}$ for the layered half-plane subjected to a concentrated horizontal force $f_{x}$ in the layer $\left(\sigma_{x y} h / f_{x}\right)$.

for material 2. A computational program for the numerical calculation of the fullfield configuration of stresses is constructed by using the explicit formulation of the solutions presented in the previous section. To generate the full-field stress contours, the series solution is truncated if the numerical calculation is to within an accuracy of $0.01 \%$. In the stress-distribution contours, short dashed lines and solid lines are used to indicate negative and positive values, respectively.

Parts $(a)$ and $(b)$ of figure 4 show the contours of stress components $\sigma_{y y}$ and $\sigma_{x y}$ in the layered half-plane due to a surface vertical concentrated force with magnitude $f_{y}$ applied at $(x, y)=(0,0)$, respectively. Both $\sigma_{y y}$ and $\sigma_{x y}$ are continuous at the interface and the inclination of the contour is due to the anisotropy of the material.

Figures 5 and 6 are stress contours, achieved by applying a horizontal concentrated force $f_{x}$ and an edge dislocation with vertical Burgers's vector $b_{y}$ in the thin layer 

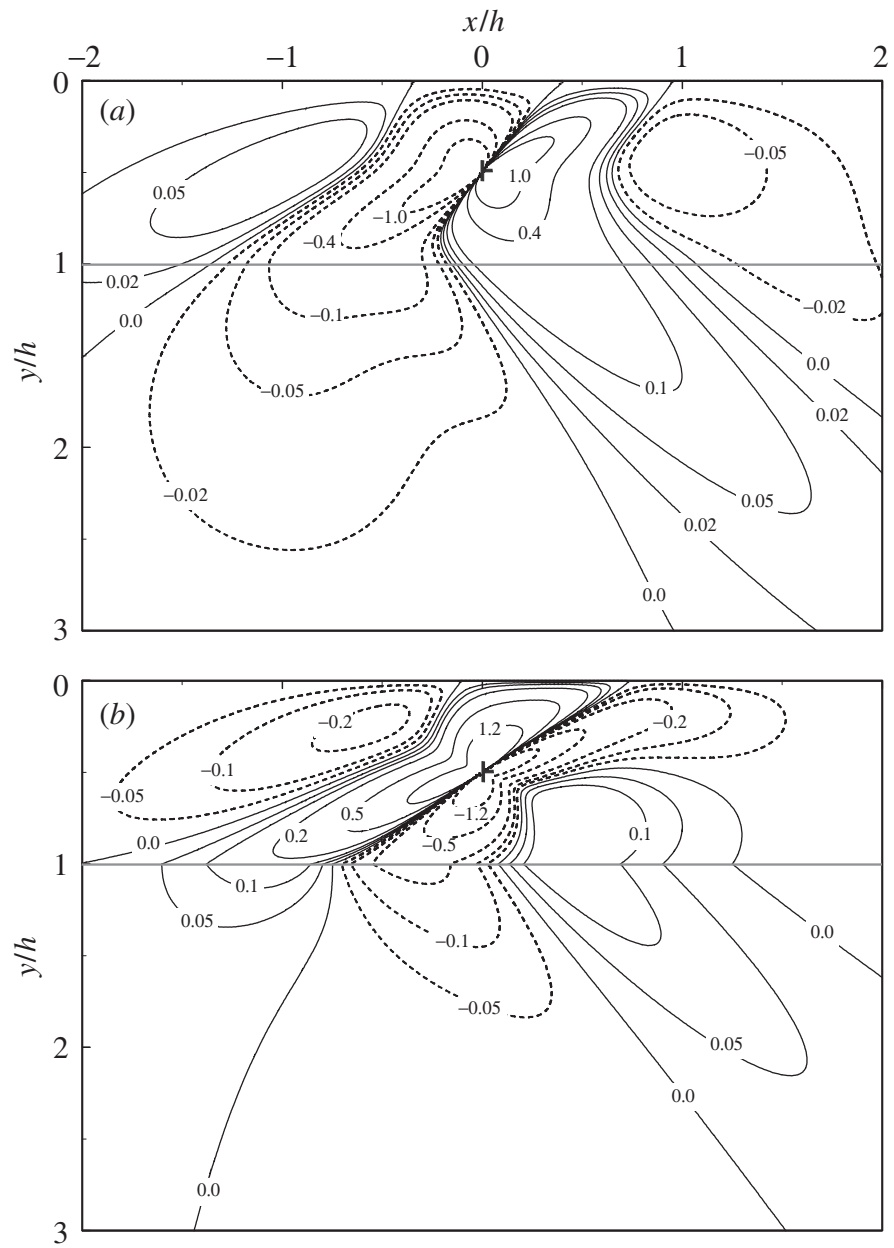

Figure 6. (a) Full-field distribution of $\sigma_{y y}$ for the layered half-plane subjected to a dislocation with Burgers's vector $b_{y}$ in the layer $\left(\sigma_{y y} s_{66} h / b_{y}\right)$. (b) Full-field distribution of $\sigma_{x y}$ for the layered half-plane subjected to a dislocation with Burgers's vector $b_{y}$ in the layer $\left(\sigma_{x y} s_{66} h / b_{y}\right)$.

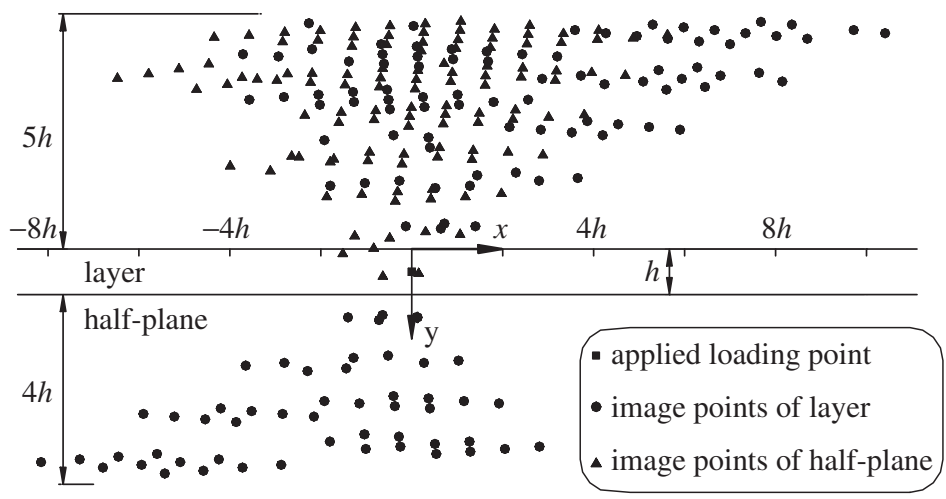

Figure 7. Image points of the layer and the half-plane for applied loadings at $(x, y)=(0,0.5 h)$.

Proc. R. Soc. Lond. A (2002) 

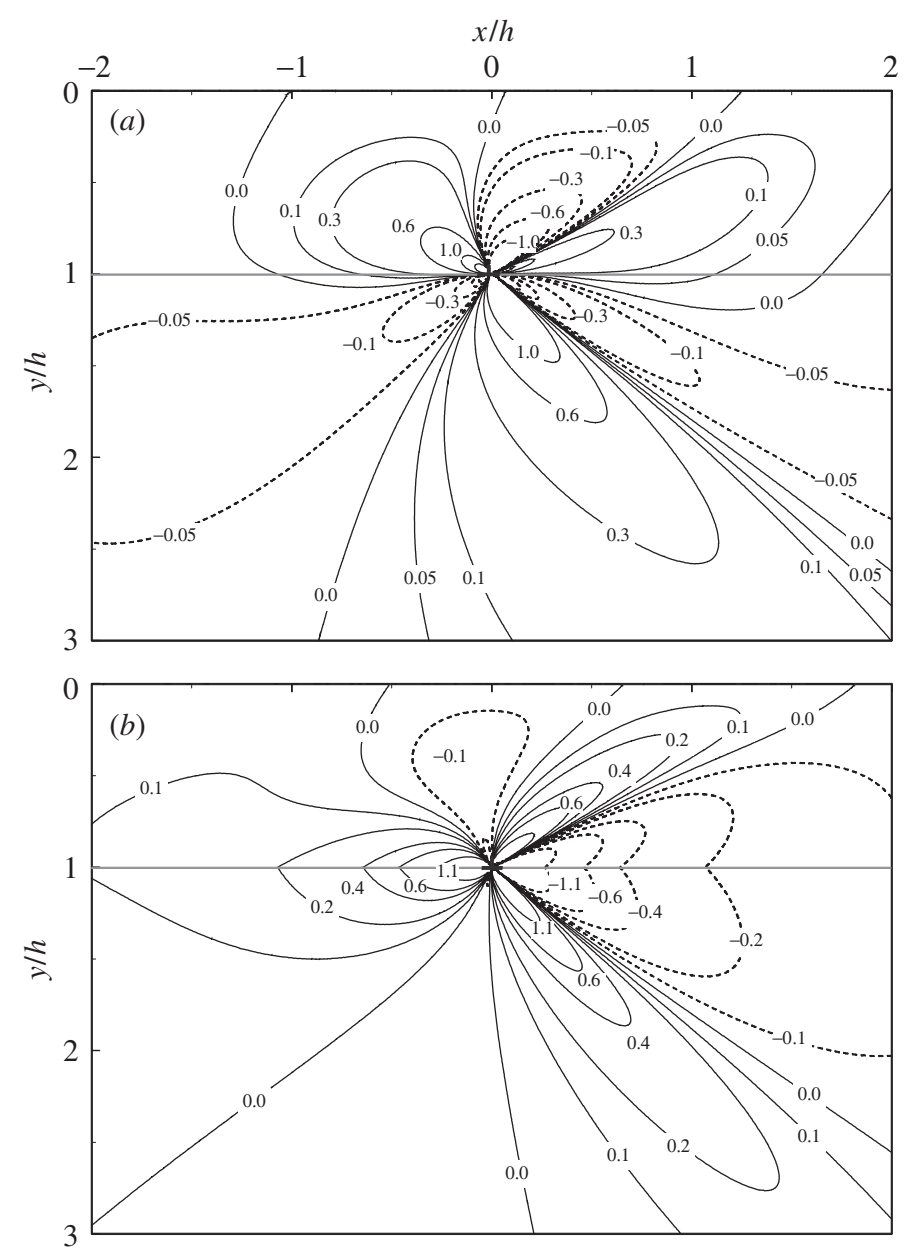

Figure 8. (a) Full-field distribution of $\sigma_{y y}$ for the layered half-plane subjected to a dislocation with Burgers's vector $b_{x}$ at the interface $\left(\sigma_{y y} s_{66} h / b_{x}\right)$. (b) Full-field distribution of $\sigma_{x y}$ for the layered half-plane subjected to a dislocation with Burgers's vector $b_{x}$ at the interface $\left(\sigma_{x y} s_{66} h / b_{x}\right)$.

at $(x, y)=(0,0.5 h)$, respectively. The stresses $\sigma_{y y}$ and $\sigma_{x y}$ are continuous at the interface and are zero on the free surface. Figure 7 shows the image points of the layer (circles, 137 points) and the half-plane (triangles, 113 points), within $-5 h \leqslant y \leqslant 5 h$, for applied loadings at $(0,0.5 h)$ in the layer, as indicated in figures 5 and 6 . We can see that the image points for the layer spread out all over the plane, except at the layer. However, the image points for the half-plane are occupied only in the region $y \leqslant h$. Usually, thousands of image points are used to obtain the result to within an accuracy of $0.01 \%$.

Figure 8 shows the stress contours of a layered half-plane subjected to an interface edge dislocation $b_{x}$ at $(x, y)=(0, h)$. Figures 9 and 10 are stress distributions of a layered half-plane subjected to a vertical concentrated force $f_{y}$ and an edge dislocation $b_{x}$ applied at $(x, y)=(0,1.3 h)$ in the half-plane, respectively.

Proc. R. Soc. Lond. A (2002) 

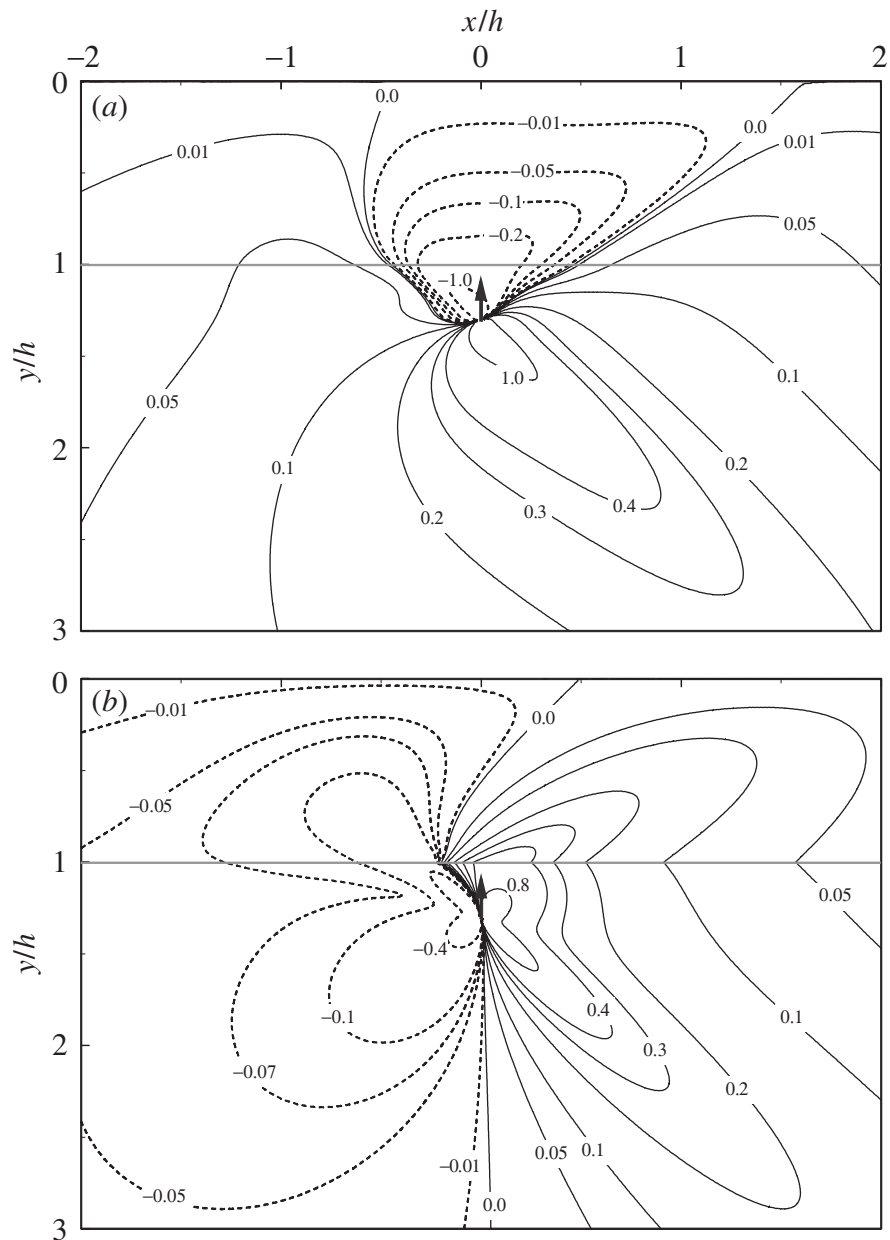

Figure 9. (a) Full-field distribution of $\sigma_{y y}$ for the layered half-plane subjected to a concentrated vertical force $f_{y}$ in the half-plane $\left(\sigma_{y y} h / f_{y y}\right)$. (b) Full-field distribution of $\sigma_{x y}$ for the layered half-plane subjected to a concentrated vertical force $f_{y}$ in the half-plane $\left(\sigma_{x y} h / f_{y}\right)$.

\section{Concluding remarks}

This study presents analytical investigations of an anisotropic layered half-plane subjected to concentrated forces and edge dislocations. By using the Fourier-transform technique and a series expansion in the transformed domain, analytical full-field solutions for stresses are obtained in an explicit closed form. The complete solution in series form consists of simple solutions for an infinite homogeneous medium with concentrated loadings. The analytical solution includes two parts: Green's function for applied loadings and an infinite number of image singularities that are induced to satisfy the boundary and interface conditions. The physical meanings of the solution can be regarded as the image method for the planar anisotropic problem. The mathematical method introduced in this study provides an automatic determination for the locations and magnitudes of all the image singularities. A computational program for calculating full-field stress distributions of the layered half-plane is easily

Proc. R. Soc. Lond. A (2002) 

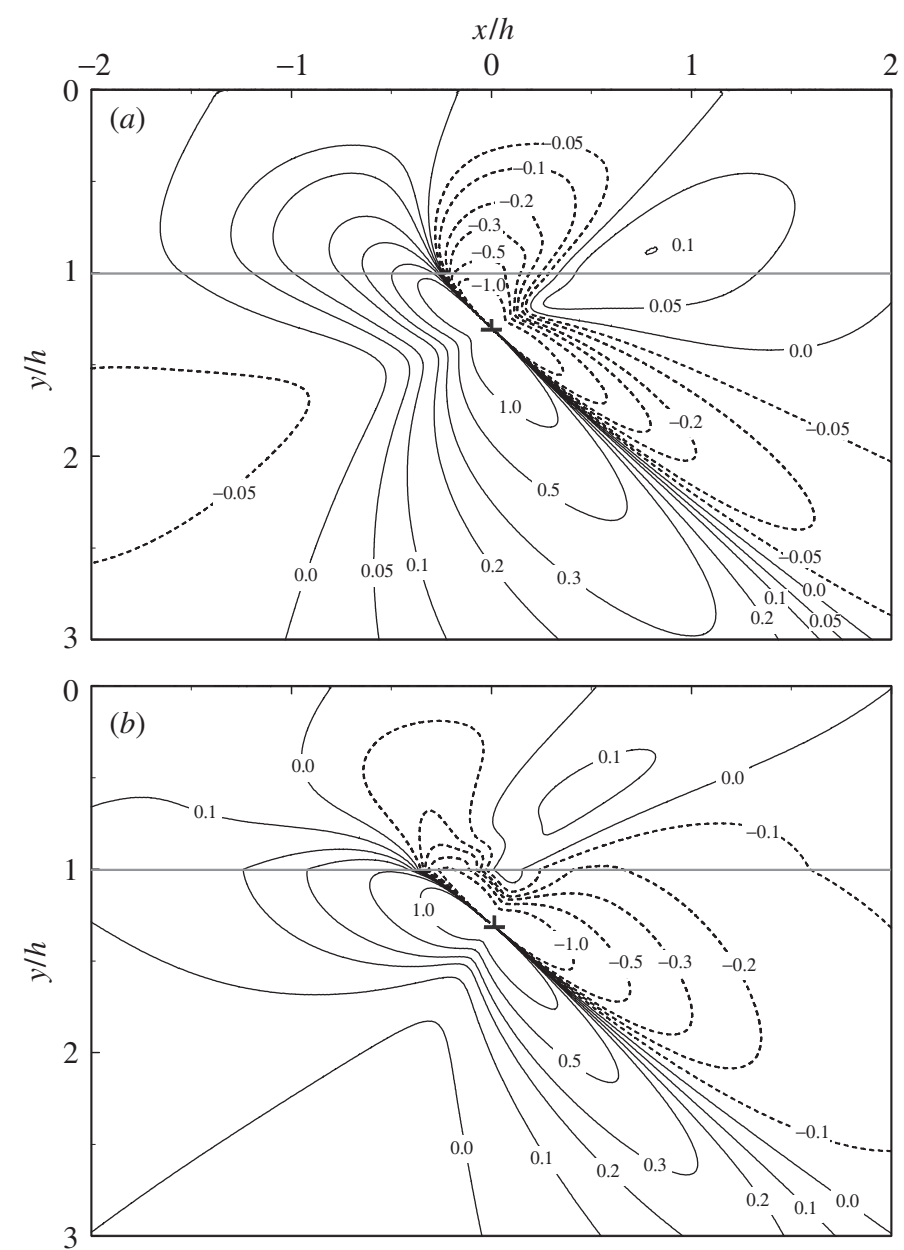

Figure 10. (a) Full-field distribution of $\sigma_{y y}$ for the layered half-plane subjected to a dislocation with Burgers's vector $b_{x}$ in the half-plane $\left(\sigma_{y y} s_{66} h / b_{x}\right)$. (b) Full-field distribution of $\sigma_{x y}$ for the layered half-plane subjected to a dislocation with Burgers's vector $b_{x}$ in the half-plane $\left(\sigma_{x y} s_{66} h / b_{x}\right)$.

constructed from the explicit formulation of the solutions. Detailed numerical results of full-field stress distributions are provided in this study for applying concentrated forces and/or edge dislocations on the boundary or in the interior of the layered half-plane.

The study of dislocations in multi-layered structures is important as a means of understanding the mechanical properties of composites and thin films. The motion of a dislocation is related to the image force or the Peach-Koehler (Peach \& Koehler 1950) force exerted on the dislocation. The image force that exerts on the dislocation is the negative of the gradient of the interaction energy that depends on the location of the dislocation. The force on the dislocation is most conveniently obtained from the Peach-Koehler formula. A method for evaluating the image forces on dislocations in the thin film is required to investigate the problem. The solutions available in the literature have been obtained for relatively simple problems, such as isotropic 
screw or edge dislocations, most of which used multiple-image analysis. However, this method is not applicable for the problem of edge dislocations in a multi-layered anisotropic medium. The explicit form of the image force acting on the edge dislocation in an anisotropic thin-film structure can be easily obtained from the results provided in this study. The image force on the edge dislocation can be calculated as the summation of force due to the stress fields of all the image singularities in the thin-film solution. A detailed investigation of the micro-mechanical behaviour for the interactions of edge dislocations in an anisotropic thin-film structure will be given in a subsequent paper.

The authors thank the National Science Council, Republic of China, and gratefully acknowledge grant no. NSC 89-2212-E002-018 to the National Taiwan University.

\section{References}

Barnett, D. M. \& Lothe, J. 1974 An image force theorem for dislocations in bicrystals. J. Phys. F 4, 1618-1635.

Buchwald, V. T. 1964 Eigenfunctions of plane elastostatics. Proc. R. Soc. Lond. A 227, 385-400.

Chiu, Y. T. \& Wu, K. C. 1998 Analysis for elastic strips under concentrated loads. ASME J. Appl. Mech. 44, 424-430.

Chou, Y. T. 1963 Planar stress field of a dislocation in anisotropic plate. J. Appl. Phys. 34, 3608-3614.

Chou, Y. T. 1966 Screw dislocations in and near lamellar inclusions. Physica Status Solidi B 17, 509-516.

Dundurs, J. \& Sendeckyj, G. P. 1965 Behavior of an edge dislocation near bimetallic interface. J. Appl. Phys. 36, 3353-3354.

Eshelby, J. D., Read, W. T. \& Shockley, W. 1953 Anisotropic elasticity with applications to dislocation theory. Acta Metall. 1, 251-259.

Gibson, R. F. 1994 Principles of composite material mechanics. McGraw-Hill.

Head, A. K. 1953 The interaction of dislocations and boundaries. Phil. Mag. 44, 92-94.

Kamat, S. V., Hirth, J. P. \& Carnahan, B. 1987 Image forces on screw dislocations in multilayer structures. Scr. Metall. 21, 1587-1592.

Lee, M. S. \& Dundurs, J. 1973 Edge dislocation in a surface layer. Int. J. Engng Sci. 11, 87-94.

Lekhnitskii, S. G. 1963 Theory of elasticity of an anisotropic elastic body. San Francisco, CA: Holden-Day.

Lin, R. L. \& Ma, C. C. 2000 Antiplane deformations for anisotropic multilayered media by using the coordinate transform method. ASME J. Appl. Mech. 67, 597-605.

Ma, C. C. \& Lin, R. L. 2001 Image singularities of Green's functions for an isotropic elastic half-plane subjected to forces and dislocations. Math. Mech. Solids 6, 503-524.

Moss, W. C. \& Hoover, W. G. 1978 Edge-dislocation displacements in a elastic strip. J. Appl. Phys. 49, 5449-5451.

Muskhelishvili, N. I. 1975 Some basic problems of the mathematical theory of elasticity, 4th edn. Groningen: Noordhoff.

Nabarro, F. R. N. \& Kostlan, E. J. 1978 The stress fields of a dislocation lying in a plate. J. Appl. Phys. 49, 5445-5448.

Öveçoğlu, M. L., Doerner, M. F. \& Nix, W. D. 1987 Elastic interactions of screw dislocations in thin films on substrates. Acta Metall. 35, 2947-2957.

Peach, M. D. \& Koehler, J. S. 1950 The force exerted on dislocations and the stress field produced by them. Phys. Rev. 80, 436-439.

Proc. R. Soc. Lond. A (2002) 
Stagni, L. \& Lizzio, R. 1986 The elastic field within an internally stressed infinite strip. Int. J. Engng Sci. 4, 471-477.

Stroh, A. N. 1958 Dislocations and cracks in anisotropic elasticity. Phil. Mag. 3, 625-646.

Suo, Z. 1990 Singularities, interfaces and cracks in dissimilar anisotropic media. Proc. R. Soc. Lond. A 427, 331-358.

Ting, T. C. T. 1992 Image singularities of Green's functions for anisotropic elastic half-spaces and bimaterials. Q. J. Mech. Appl. Math. 45, 119-139.

Ting, T. C. T. 1996 Anisotropic elasticity: theory and applications. Oxford University Press.

Ting, T. C. T. \& Barnett, D. M. 1993 Image force on line dislocations in anisotropic elastic half-spaces with a fixed boundary. Int. J. Solids Struct. 30, 92-94.

Tullini, N. \& Savoia, M. 1999 Elasticity interior solution for orthotropic strips and the accuracy of beam theories. ASME J. Appl. Mech. 66, 368-373.

Weeks, R., Dundurs, J. \& Stippes, M. 1968 Exact analysis of an edge dislocation near a surface layer. Int. J. Engng Sci. 6, 365-372.

Wu, K. C. \& Chiu, Y. T. 1995 The elastic fields of a dislocations in an anisotropic strip. Int. J. Solids Struct. 32, 543-552.

Proc. R. Soc. Lond. A (2002) 